\title{
Scenario-based approach adopted in the ELECTRA project for deriving innovative control room functionality
}

Marinelli, Mattia; Heussen, Kai; Prostejovsky, Alexander Maria; Bindner, Henrik W.; Catterson, Victoria M.; Merino, Julia; Tornelli, Carlo

\section{Published in:}

Cired - Open Access Proceedings Journal

Link to article, DOI:

10.1049/oap-cired.2017.0613

Publication date:

2017

Document Version

Publisher's PDF, also known as Version of record

Link back to DTU Orbit

Citation (APA):

Marinelli, M., Heussen, K., Prostejovsky, A. M., Bindner, H. W., Catterson, V. M., Merino, J., \& Tornelli, C. (2017). Scenario-based approach adopted in the ELECTRA project for deriving innovative control room functionality. Cired - Open Access Proceedings Journal, 2017(1), 1450 - 1453. https://doi.org/10.1049/oapcired.2017.0613

\section{General rights}

Copyright and moral rights for the publications made accessible in the public portal are retained by the authors and/or other copyright owners and it is a condition of accessing publications that users recognise and abide by the legal requirements associated with these rights.

- Users may download and print one copy of any publication from the public portal for the purpose of private study or research.

- You may not further distribute the material or use it for any profit-making activity or commercial gain

- You may freely distribute the URL identifying the publication in the public portal 


\title{
Scenario-based approach adopted in the ELECTRA project for deriving innovative control room functionality
}

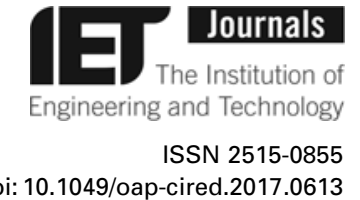
doi: 10.1049/oap-cired.2017.0613 www.ietdl.org

\author{
Mattia Marinelli ${ }^{凶}$, Kai Heussen ${ }^{1}$, Alexander Prostejovsky ${ }^{1}$, \\ Henrik W. Bindner ${ }^{1}$, Victoria M. Catterson ${ }^{2}$, Julia Merino ${ }^{3}$, \\ Carlo Tornelli ${ }^{4}$ \\ ${ }^{1}$ Department of Electrical Engineering, Technical University of Denmark (DTU), Roskilde, \\ Denmark \\ ${ }^{2}$ Institute for Energy and Environment, University of Strathclyde, Glasgow, UK \\ ${ }^{3}$ TECNALIA, Gipuzkoa, Spain \\ ${ }^{4} R S E$, Milano, Italy \\ 凶-mail: matm@elektro.dtu.dk
}

\begin{abstract}
Here, the authors analyse the operator point of view of the Web-of-Cells concept defined in the EU project ELECTRA, by identifying operator tasks into the supervision of a highly automated power system, and the information requirements to facilitate appropriate operator situation awareness. The study outlines the methodology adopted, which is based on the cognitive work analysis framework, to provide an overview of the most interesting scenarios and to summarise the requirements analysis results. In order to derive required control room functionality, a set of relevant control room scenarios have been identified based on the Web-of-Cells control concept. The authors considered scenarios that challenge traditional control schemes, scenarios that caused major failures (i.e. blackouts), and scenarios that can be expected to appear in the future. For each scenario, information concerning network layout, triggering events, physical constraints, manually/automatic operations, operators' tasks, and relevant analytics have been analysed.
\end{abstract}

\section{Introduction}

With the increased flexibility within the power system, system-wide adoption of dynamic ratings, pervasive control and automation, increasing market influence etc., it will remain essential to have control room operators aware of the system state and of potential threats, and informed of the actions of automatic control systems. Considering these encompassing alterations to the system behaviour and functions, it is recognised that significantly improved information and visualisation tools are fundamental for future control rooms [1,2].

The approach adopted in the EU ELECTRA Integrated Research Programme to deal with power system control is based on the power transmission and distribution system in the future as a highly automated web of subsystems, called cells. The cell operators (COs) have to ensure that the cells maintain their individual agreed schedules and reserves. For control purposes, a $\mathrm{CO}$ has to act on the inner resources of its own cell and can also cooperate with other COs, in particular with the neighbouring cells' COs, so that the whole power system, the Web-of-Cells (WoC), is stable, secure, and reliable [3].

The control room perspective entails that the overall operation of a WoC coordinated power system has to be taken into account. Whereas software/control functions are designed to act on specific subobjectives and stability problems from the $\mathrm{CO}$ perspective, an overview of the overall system state has to be addressed.

This paper intends to outline the methodology adopted, which is based on the cognitive work analysis (CWA) framework [4], to provide an overview of the most interesting scenarios and to summarise the requirements analysis results [5].

\section{Methodology adopted}

The control room perspective entails that the 'big picture' of a WoC coordinated power system operation has to be taken into account.
Whereas software/control solutions are designed with separate objectives and stability problems in mind, in the CO perspective, an overview of the overall system state has to be addressed. In view of the ELECTRA WoC concept, the operator task is to supervise a highly automated power system's operation and have the option and capacity to intervene if necessary [6].

The operator support functions provided in the control room can be divided into three aspects:

- System monitoring: operator situational awareness (SA); can you evaluate what is critical right now?

- Supervisory control and interventions: offer input for operator to adjust system state.

- Decision support: help operator identifying the right intervention.

The design of visualisation and decision support systems for supervisory control of increasingly automated systems is a challenge, as increasing automation does not necessarily reduce the cognitive effort for operators, and in particular in critical situations, more automated systems have been reported to cause a higher strain on an operator's decision-making capacity [7]. In order to define detailed requirements for control room solutions, the designer thus has to understand what constitutes relevant information to be presented to the operator.

To characterise these requirements for further technical analysis and design, the main outcome of a further analysis is the identification and prioritisation of this relevant information. To be able to formulate this information, however, we need to provide a meaningful context of description and analysis. A systematic approach to such requirements analysis for human machine interactions has been developed as Cognitive Systems Engineering. On this background, an analysis methodology called 'CWA' has been applied [8]. CWA offers a stepwise methodology for systematically identifying and constructing a knowledge context in which this relevant information can be described. 
Given the speculative and anticipatory setting of the ELECTRA work, these requirements are hard to identify directly from interviews with operators, but can be derived and revisited from a scenario analysis with domain experts instead.

The CWA analysis methodology has been summarised as follows:

[...] the overall approach [consists] of five interrelated phases of modelling:

1. The work domain - purpose and structure of the system being controlled.

2. Activity or control task analysis - what needs to be done in the work domain.

3. Mental strategies - the mechanisms by which control tasks can be achieved.

4. Social organisation - who carries out the work and how it is shared.

5. Worker competencies - the set of constraints associated with the workers themselves.

In principle there are many specific modelling techniques that could serve for each of these phases. [...] The CWA approach therefore provides an interrelated set of methodologies within which these differing aspects of a system can be mapped, examined and analysed. For example, CWA provides a means by which decision making within an environment can be associated with system goals and cognitive skills.

Adopting this methodology for the purpose of our analysis, the first step is therefore to describe how the system (the work domain) 'looks' (presents itself) from the operator point of view: to describe the operating objectives, power system, and control functions at several levels of detail. In common CWA practice, the abstraction-decomposition (Rasmussen's abstraction hierarchy [9]) space is applied for Step 1 , and a hierarchically organised analysis of the operator's decision-making (Rasmussen's decision-ladder [9]) is employed for Step 2.

A contribution of the ELECTRA project has been to demonstrate how the presently well-adopted Use Case methodology can be employed to provide the type of information required for Step 1: by formulating the required control structures and functions for the WoC concept in both abstract form (high-level use cases) and more detailed technical form (detailed use cases), a clear decomposition of the work domain has been formulated [10].

To address Step 2 of the CWA methodology (control task analysis), critical operation scenarios, defined 'Control Room Scenarios', have been identified and extensively analysed [5]

The elements identified in each scenario are listed below and can be related to CWA and SA contexts:

(i) control room scenario name;

(ii) network layout; initial conditions and schedule (domain context);

(iii) categorisation of scenario (characterisation of scenario assumptions);

(iv) involved operators and coordination among operators (social context);

(v) triggering event (starting point of an event sequence; trigger in decision ladder);

(vi) relevant physical and operational constraints (interpretation and prioritisation of system state; information analysis/ comprehension);

(vii) initially/automatically affected control loops (use cases; automatic response/automation);

(viii) grid visualisation (context representation/information acquisition);

(ix) operator first task - awareness of system change of state and operating state (SA); (x) operator second task - decision/action (Level 2 SA with decision and action);

(xi) operator third task - optimisation (Level $3 \mathrm{SA}$; operator cooperation with decision support system);

(xii) relevant analytics (e.g. available control capacity from flexibility resources).

It can be observed that pragmatic simplifications have been performed in the formulation of this method. These simplifications have been motivated from the perspective that a pragmatic analysis that is approachable for the project participants will generate more relevant results than a rigorous analytical approach that has the risk of alienating the participants. As Endsley and Garland [7] report, 'the problem of meaning [ought to] be tackled head on': the chosen formulation of the SA and CWA methodology for scenario analysis offered more significance for the project participants than a pure approach.

This pragmatic approach has been further pursued later in the work, where the control tasks were analysed in terms of a sequence analysis, accounting both for required decisions and analytics and the required information exchange. This control scenario (control task) analysis is further deepened by a sequence analysis that includes both operator and control system information and decision flows. Such an annotated sequence diagram therefore addresses aspects of Steps 3 and 4 in the CWA methodology outlined above. An analysis of 'worker competencies' (CWA Step 5 ) has not been considered feasible to address analytically at this stage.

\section{Scenario examples}

Three main drivers are identified for defining the scenarios: scenarios that challenge traditional control schemes, scenarios that caused major failures (i.e. blackouts), and scenarios that will happen in the future (not experienced yet). The interested reader may get the full picture in [5], while in the present paper, two particularly interesting scenarios are reported, providing the control room perspective on the activation of two use cases defined in the project, namely balance steering control (BSC) and post-primary voltage control (PPVC) [11]. For each scenario, a brief description is provided along with the control room perspective.

\subsection{Inter-cell loop flows}

The electricity market determines solutions for electric power flows based on the market prices and subsequent bids of participants in the market. In the WoC concept, automated BSC determines flow schedules based on the market solution, as these contracted flows do not necessarily follow physical occurrences and the actual flows may differ in a meshed grid. Loop flows are therefore defined as the deviation of the actual power flows from their scheduled values as described in [12].

Various reasons can cause loop flows, which may not be harmful per se but may lead to problems when not considered. The two main factors are insufficient price signals, where market prices do not reflect physical realities and constraints, as well as increasing energy imbalances due to volatile renewable energy resources that are increasingly deployed in the grid.

Fig. 1 illustrates various different flow situations in a meshed grid. The scheduled flow within market participant (cell) A as part of the market solution is shown in Fig. $1 a$, whereas Fig. $1 b$ shows the actual emerging physical flow through neighbouring cells. The resulting unscheduled flow in Fig. $1 c$ is the difference between Figs. $1 a$ and $b$. Loop flows are the parts of the scheduled flow that take alternative paths as indicated in Fig. $1 d$. Inter-cell flows are essentially the same as within a cell, only that now the scheduled power flow crosses cell borders via tie-lines as demonstrated in Fig. $1 e$ between cells $\mathrm{A}$ and $\mathrm{D}$. The resulting inter-cell loop flow through cells $\mathrm{B}$ and $\mathrm{C}$ is given in Fig. 1e. 


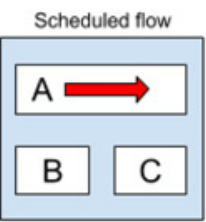

a

Loop flow

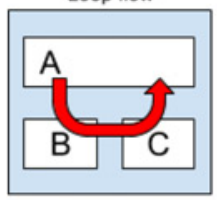

$d$

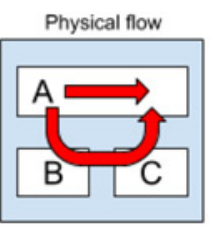

$b$

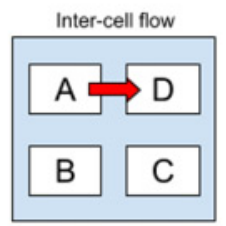

e
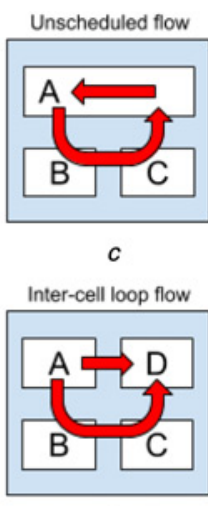

$f$
Fig. 1 Terms explaining inter-cell loop flows

Problems related to loop flows are operational security, where unhandled flows can potentially lead to blackouts, reduced economic and physical efficiency, and increased overall costs due to contract violations, among others. For this reason, several mitigating means have been established that allow the control of flows to a certain extent, such as phase-shifting transformers, series and shunt compensators etc. In addition, synchronous machines and HVDC links can be utilised to alter loop flows.

Within ELECTRA, we target for an automated control system that realises the best possible solution for the scheduled flows under the given physical constraints. The BSC calculates a physically feasible solution to the desired market operating points, which is then realised by the BSC. Real-time monitoring of all production and consumption together with topological information allows the control system to react immediately on changing conditions and steer the grid back to its optimal operating point using available flexibility resources. Deviations from scheduled flows are therefore minimised. The remaining permanent deviations should ideally be fed back into the market and reflected in the price signals in order to mitigate unwanted flows after the next market clearing.

From a control room operator's point of view, significant deviations from the scheduled flows that do not cease after balancing actions indicate problems in the automated control system. These problems may have different causes, such as poor grid models the control is operating on, corrupted live data streams within and from other cells, falsely reported operational states of generators/breakers/etc., among many others that are outside of the normal operational state. It is therefore the operator's task to interpret unscheduled power flows for their potential causes and take appropriate countermeasures. Sticking to the mentioned examples, these measures could be contacting other COs to update the grid models, checking the state of the IT network, sending technicians on site to observe the actual state of devices etc. In the case of imminent tie-line overloading, manual override of generators and loads near the affected line may be necessary to relieve stress. In all cases, the decision support system should suggest possible causes and solutions for the unscheduled loop flows to the operator, who evaluates them and (if applicable) accepts or refuses the suggested mitigating action.

The premise for the inter-cell loop flow scenario is that the BSC comes up with tie-line flow schedules that are physically feasible under normal operating conditions, which are maintained by automated controllers. Any significant deviations from the schedule therefore indicate problems that are not handled automatically, for which reason the decision support system should help the CO to identify the cause of the problem and potentially suggest solutions.

Fig. 2 illustrates the general scheme for this scenario. The loop flow algorithm, executed within the cell control centre, observes the flows in the power grid via the Distributed SCADA system, which acquires live data from the WoC. If a significant deviation from the scheduled flows is detected, the algorithm enquires

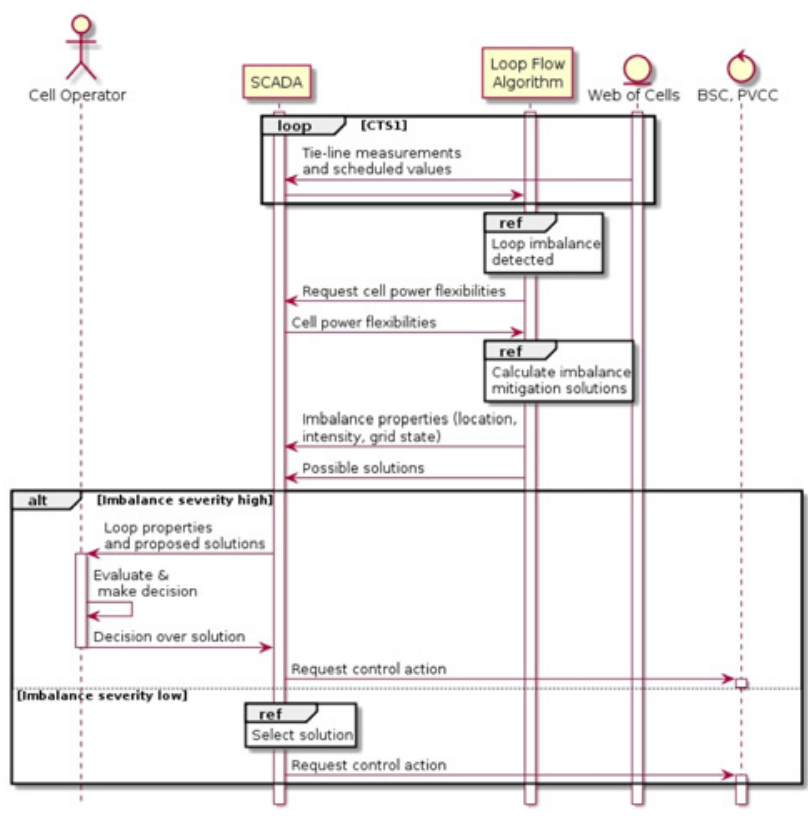

Fig. 2 Sequence diagram for handling inter-cell loop flows

additional grid state information in order to derive the problem severity and mitigating solutions. If the severity is low, the control system selects the most appropriate solution and hands it over to the BSC and/or PPVC as an additional control objective. In the case of high problem severity, however, the loop properties and proposed solutions are presented to the human operator, who then has to interpret and evaluate the facts and request appropriate countermeasures, if applicable.

\subsection{Proactive operation of the voltage control}

In the analysis of future provision of ancillary services within ELECTRA, two control layers were identified for the fulfilment of voltage control goals: primary voltage control (PVC) and post primary voltage control (PPVC). Since the PVC will remain similar to the current practices - even new devices will take action -, the PPVC will involve radical changes for the WoC control, monitoring, and operational procedures.

This scenario focuses on the novel strategies to be accomplished by the $\mathrm{CO}$ in the control room for the proactive operation of the PPVC. The PPVC, working in proactive mode, is intended to reduce the number of triggers of the PVCs by anticipating the voltage deviations in the nodes. Based on short-term forecasts and in $15 \mathrm{~min}$ intervals analysis, the $\mathrm{CO}$ compares the real-time voltage measurements with the expected voltages in the nodes. This way it is possible to correct the voltage deviations in advance, avoiding unnecessary trips of the PVC.

For the correct operation of the proactive PPVC, the operator in the control room must have a complete snapshot of:

- the measurements in the $\mathrm{WoC}$, including the voltages in the nodes, the tie-line power flows, the available PPVC reserves etc.,

- the static information concerning the allowable voltage ranges in the nodes, the capacities of the lines, or the voltage safe bands for the nodes.

However, it is also critical to have reliable forecasts (rooted on generation/load profiles) of the short-term voltages in the nodes. In the case of any bad measurement that could lead to the wrong operation or even the windup of the control system, the PVC will act as the automatic backup mechanism.

Summarising the tasks of the operator, the steps to follow in the operational procedure of the proactive PPVC would be the following: 


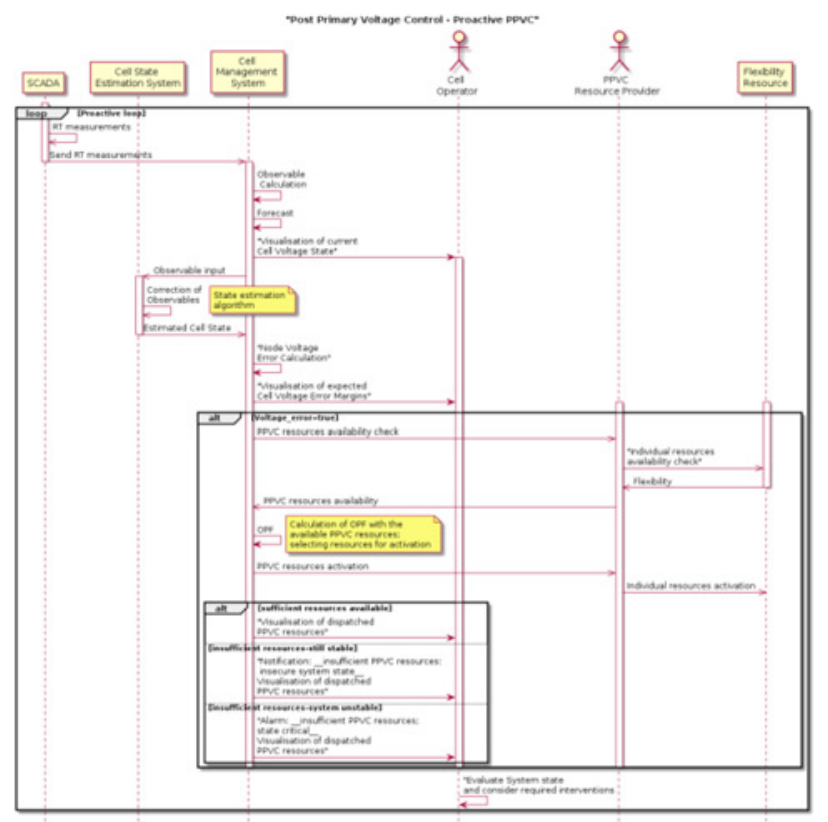

Fig. 3 Sequence diagram for proactive voltage control

- monitoring of the RMS voltage and the voltage deviation in the nodes,

- calculation of voltage short-term forecasts,

- estimation of the cell state for the following $15 \mathrm{~min}$ using real-time measurements, observables, and short-term forecasts,

- detection of unacceptable deviation of the voltages in any node with regard to the schedule for the next $15 \mathrm{~min}$,

- proactive activation of the PPVC reserves in accordance.

In Fig. 3, the detailed sequence diagram for the proactive operation of the PPVC is shown. In it, the main relationships between actors and elements involved are represented by means of the information exchanges. The external loop contains the processes and information exchanges that belong to the continuous operation of the PPVC in a time horizon of $15 \mathrm{~min}$. The inner loop actions only take place in the case of a voltage deviation from the safe band which would imply the recalculation of new voltage set-points.

The SCADA system managed by the control room operator is continuously supervising the operation of the WoC. It registers the measurements, the observables (original ones and those corrected by the cell state estimation) and also contains the static or semi-static information of the network (topology, breakers' status etc.). On a fully automated basis, the control room operator must be able to:

- compare the estimated cell state for the 15 min horizon window with the limit values;

- detect the abnormal condition in any node and launch a proactive OPF process (if necessary);

- notify the $\mathrm{CO}$ and suggest solutions, given the resources available;
- send the new set-points to the local controllers in order to keep the voltages within the limits established to optimise the system's power flows.

\section{Conclusions}

The scenarios have been compiled to create an overview of possible situations in future power systems arising under the ELECTRA WoC approach. The objective of outlining these scenarios has been to identify specific requirements for visualisation, analytics, and decision support. A systematic approach to such requirements analysis for human machine interactions has been developed as Cognitive Systems Engineering. On this background, an analysis methodology called 'CWA' has been applied, which offers a stepwise methodology for systematically identifying and constructing a knowledge context in which this relevant information has been described.

Two scenarios, namely 'inter-cell loop flow' and 'proactive operation of post-primary voltage control', have been described in order to clarify the methodology adopted. A cross-sectional analysis of the above scenarios to reflect their coverage with respect to possible future situations, network levels and topologies, and the WoC automatic control systems is reported and subsequently requirements have been drawn.

\section{Acknowledgment}

The work in this paper was supported by the European Commission, under the FP7 project ELECTRA (grant no: 609687).

\section{References}

1 Sand, K.: 'Next generation control centres - state of art and future scenarios' Norwegian University of Science and Technology (NTNU), Trondheim, Technical Report 1/2012, 2012

2 Rezkalla, M., Heussen, K., Marinelli, M., et al.: 'Identification of requirements for distribution management systems in the smart grid context'. 2015 Proc. of the 50th Int. Universities Power Engineering Conf. (UPEC), 2015, pp. 1-6

3 Martini, L., Radaelli, L., Brunner, H., et al.: 'ELECTRA IRP approach to voltage and frequency control for future power systems with high DER penetrations'. 23rd Int. Conf. on Electricity Distribution CIRED, 2015, pp. 1-4

4 Rasmussen, J., Pejtersen, A., Goodstein, L.P.: 'Cognitive systems engineering' (Wiley, New York, 1994)

5 Marinelli, M., Heussen, K., Strasser, T., et al.: 'Demonstration of visualization techniques for the control room engineer in 2030'. ELECTRA Deliverable D8.1. WP8: Future Control Room Functionality, 2017

6 Guttromson, R.T., Greitzer, F.L., Paget, M.L., et al.: 'Human factors for situation assessment in power grid operations'. Pacific Northwest National Laboratory Report - PNNL16780, 2007

7 Endsley, M.R., Garland, D. J.: 'Theoretical underpinnings of situation awareness: a critical review'. Situation Awareness Analysis and Measurement, 2000, pp. 3-32

8 Vicente, K.J.: ‘Cognitive work analysis. Mahwah' (Lawrence Erlbaum Associates, NJ, 1999)

9 Rasmussen, J., Pejtersen, A. M., Goodstein, L. P.: 'Cognitive systems engineering' (Wiley, New York, 1994)

10 Endsley, M.R., Connors, E.S.: 'Situation awareness: state of the art'. Power and Energy Society General Meeting - Conversion and Delivery of Electrical Energy in the 21 st Century, 2008 IEEE. IEEE, 2008

11 Caerts, C., D'hulst, R., De Breucker, S., et al.: 'Specification of smart grids high level functional architecture for frequency and voltage control'. ELECTRA Deliverable D3.1. WP3 Scenarios and Case Studies for Future Power System Operation, 2015

12 Marinelli, M., Pertl, M., Rezkalla, M., et al.: 'Functional description of the monitoring and observability detailed concepts for the Pan-European control schemes'. ELECTRA Deliverable D5.4. WP5: Increased Observability, 2017 